\title{
Phosphine Dissociation on the Si(001) Surface
}

\author{
H. F. Wilson, ${ }^{1}$ O. Warschkow, ${ }^{1}$ N. A. Marks, ${ }^{1}$ S. R. Schofield, ${ }^{2,3}$ N. J. Curson, ${ }^{3}$ P.V. Smith, ${ }^{2}$ M.W. Radny, ${ }^{2}$ \\ D. R. McKenzie, ${ }^{1}$ and M. Y. Simmons ${ }^{3}$ \\ ${ }^{1}$ Centre for Quantum Computer Technology, School of Physics, The University of Sydney, Sydney 2006, NSW Australia \\ ${ }^{2}$ School of Mathematical and Physical Sciences, The University of Newcastle, Callaghan 2308, NSW Australia \\ ${ }^{3}$ Centre for Quantum Computer Technology, School of Physics, University of New South Wales, Sydney 2052, NSW, Australia \\ (Received 11 June 2004; published 23 November 2004)
}

Density functional calculations are performed to identify features observed in STM experiments after phosphine $\left(\mathrm{PH}_{3}\right)$ dosing of the $\mathrm{Si}(001)$ surface. On the basis of a comprehensive survey of possible structures, energetics, and simulated STM images, three prominent STM features are assigned to structures containing surface bound $\mathrm{PH}_{2}, \mathrm{PH}$, and $\mathrm{P}$, respectively. Collectively, the assigned features outline for the first time a detailed mechanism of $\mathrm{PH}_{3}$ dissociation and $\mathrm{P}$ incorporation on $\mathrm{Si}(001)$.

DOI: 10.1103/PhysRevLett.93.226102

With the continued downscaling of electronic components to the atomic scale, a number of novel devices become possible, including quantum cellular automata [1], single electron transistors [2], and quantum computers [3]. The fabrication of such devices invariably requires scanning probe techniques and an atomic-level understanding of doping mechanisms and the intermediate species involved. In the case of the silicon quantum computer proposed by Kane [3], the phosphorus qubits are fabricated using $\mathrm{PH}_{3}$ molecules precisely placed using scanning tunneling microscopy (STM) [4]. It is well established that $\mathrm{PH}_{3}$ adsorbs dissociatively on the $\mathrm{Si}(001)$ surface at room temperature; however, much controversy surrounds the numerous intermediate species observed in STM experiments. Of particular importance is a bright protusion centered atop a $\mathrm{Si}$-Si-dimer as seen in numerous STM studies [4-10]. No fewer than four different structures have been advanced for this feature but none satisfactorily explain the experimental data. This Letter, drawing on an extensive ab initio survey of possible $\mathrm{PH}_{3}$ dissociation products, identifies this and two other prominent STM features as $\mathrm{PH}, \mathrm{PH}_{2}$, and $\mathrm{P}$ species, respectively. All three structures are fully consistent with STM data. Moreover, experimentally observed transitions between these three species are consistent with (and explained by) the relative energetics of the calculated species. Collectively, these structures outline a mechanism for $\mathrm{PH}_{3}$ dissociation and $\mathrm{P}$ incorporation on the $\mathrm{Si}(001)$ surface.

Figure 1 shows representative STM images following low-dose $(<0.001 \mathrm{~L}) \mathrm{PH}_{3}$ exposure of the $\mathrm{Si}(001)$ surface at room temperature. These images are selected from a comprehensive experimental study, involving hundreds of dosing experiments, the details of which will be presented in a separate publication [11]. In this Letter we present a theoretical assignment of three common features, all of which are routinely observed after $\mathrm{PH}_{3}$ dosing: the asymmetric, centered, and U-shaped features as labeled in Fig. 1(a). The asymmetric feature, shown
PACS numbers: 68.35.-p, 68.37.Ef, 68.47.Fg, 73.20.At

in high-resolution filled- and empty-state images in Figs. 1(b) and 1(e) has previously been assigned as $\mathrm{PH}_{2}$ and $\mathrm{H}$ bound to the same dimer [5]. Four different interpretations have been offered to explain the centered feature shown in Figs. 1(c) and 1(f) that sits symmetrically on a $\mathrm{Si}$ dimer. Initially, it was proposed $[6,12]$ that a $\mathrm{PH}_{3}$ molecule centered atop a $\mathrm{Si}$-Si dimer gives rise to this feature. However, subsequent $a b$ initio calculations $[8,13,14]$ have shown that such a structure is unstable. Kipp et al. [8] suggested that the feature is a P-P dimer; however, this has been discounted by experimental observations [7,10]. The centered $\mathrm{PH}_{2}$ model of Lin et al. [9] is discounted in this work on energetic grounds. Finally,
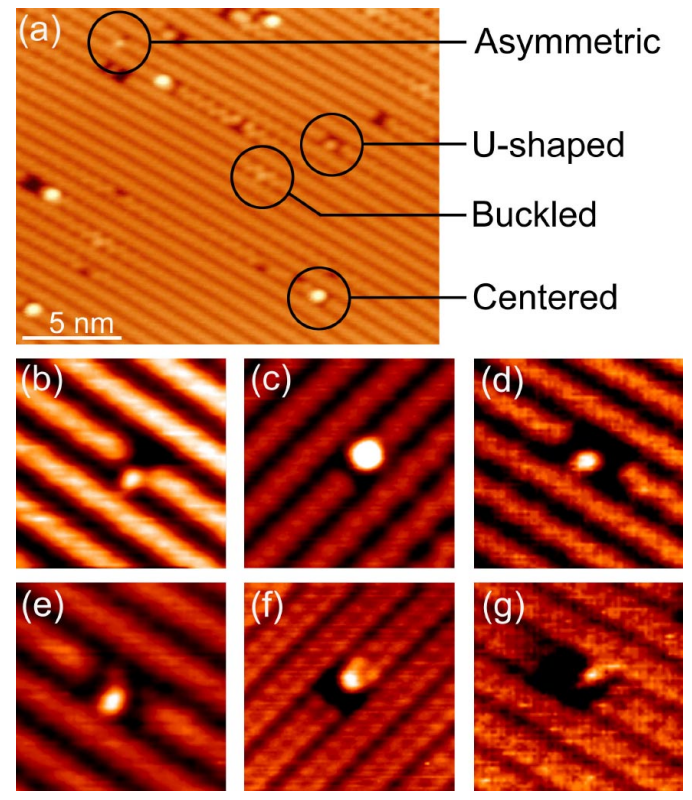

FIG. 1 (color online). (a) Filled-state STM image of Si(001) after room-temperature dosing with $\mathrm{PH}_{3}$. (b)-(d) Highresolution filled-state images of the asymmetric, centered, and U-shaped features. (e)-(g) Empty-state images of the same. STM images acquired with $-1.6 \mathrm{~V} /+1.2 \mathrm{~V}$ and $0.1 \mathrm{nA}[5,10,11]$. 
Miotto et al. [14] suggested that the centered feature is the dissociation product $\mathrm{PH}_{2}+\mathrm{H}$ on a single dimer. However, their calculated STM image was asymmetric whereas the experimental images are unambigously centered on the dimer. The U-shaped feature, shown in filled- and emptystate images in Figs. 1(d) and 1(g) has not previously been reported. The buckled feature in Fig. 1(a) does not contain phosphorus [11] and is therefore not a part of this study.

Common to all the results listed above is that extensive experimental data have not permitted an unambiguous assignment of the STM features. Detailed quantum chemical calculations reported in this Letter can assist in this task on two levels: (a) It provides an unbiased survey of all conceivable dissociation intermediates (i.e., structures containing one $\mathrm{PH}_{x}(x=0,1,2,3)$ and $3-x \mathrm{H}$ atoms bound to the surface). This identifies the structures of highest stability that are the most likely intermediates along a thermodynamically-driven dissociation path. (b) Using STM image simulation, structures in this limited set of favored intermediates are matched with experimental STM features.

All calculations were performed using first-principles density functional methods. The bulk of the structure survey was undertaken using the CPMD package [15] (Goedecker pseudopotentials, a plane-wave cutoff of 18 Ry, BLYP exchange-correlation functional, and $\Gamma$-point sampling). The surface was represented using a five-layer slab model in a $4 \times 4$ cell containing eight dimers in two rows. In order to confirm that computational details such as cell size, k-point sampling, and choice of pseudopotential do not impact on our findings, supporting calculations on a smaller $2 \times 4$ cell were performed using both CPMD and the VASP (Vienna ab initio simulation package) software [16] (PW91 functional, four-layer slab model, Vanderbilt pseudopotentials, 12.8 Ry cutoff, four special $\mathbf{k}$ points). For selected structures, energies were also calculated using the Gaussian 03 software [17] and a three-dimer $\mathrm{Si}_{21} \mathrm{H}_{20}+\mathrm{PH}_{3}$ cluster model [B3LYP functional, mixed 6-311++G(d,p), and LANL2DZ basis sets]. For the structures relevant to this discussion, all methods agree on the general energetic stability and we will in the following refer to the results of the CPMD $(4 \times 4)$-slab calculations unless otherwise stated. Structures in our survey were generated by distributing the $\mathrm{PH}_{x}$ unit $(x=0,1,2,3)$ and $3-x$ hydrogen atoms over available binding sites on the $\mathrm{Si}(001)$ surface: dimer end $\left(\mathrm{PH}_{x}\right.$ or $\mathrm{H}$ bonded to one $\mathrm{Si}$ atom of a Si-Si dimer), dimer bridge $\left(\mathrm{PH}_{x}\right.$ sits symmetrically above a dimer and is bonded to both $\mathrm{Si}$ ), and end bridge $\left(\mathrm{PH}_{x}\right.$ bonded across adjacent dimers). An additional set of structures contains phosphorus incorporated into the surface, replacing a $\mathrm{Si}$ atom in a surface dimer. The ejected $\mathrm{Si}$ atom is placed onto the surface as an adatom.

Figure 2 gives an energy overview for all stable structures in our survey. Every structure is represented by a horizontal bar, placed into the figure according to the energy of the structure and the type of $\mathrm{PH}_{x}$ unit $\left(\mathrm{PH}_{3}\right.$,

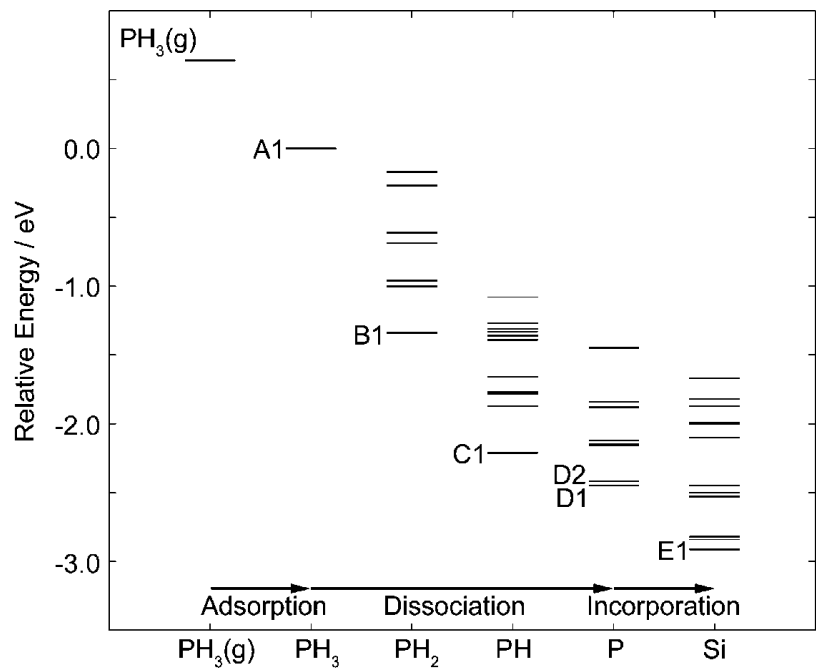

FIG. 2. Overview of computed energies for structures considered in our survey of stable $\mathrm{PH}_{3}$ adsorption and dissociation products on the $\mathrm{Si}(001)$ surface. Structures are ordered on the horizontal axis according to $\mathrm{PH}_{x}$ fragment $(x=3,2,1,0)$, indicating the degree of dissociation. Low energy configurations along the dissociation series from left to right are labeled; the corresponding structures are shown in Fig. 3.

$\mathrm{PH}_{2}, \mathrm{PH}$, and $\mathrm{P}$ ) it contains. Gas-phase $\mathrm{PH}_{3}$ and structures containing $\mathrm{Si}$ adatoms are placed into separate columns. In this arrangement, Fig. 2 provides two important insights: First, $\mathrm{PH}_{3}$ adsorption, progressive dissociation, and finally incorporation of $\mathrm{P}$ into the surface leads to increasing energetic stability. Second, in each of the $\mathrm{PH}_{x}$ sets, one or two structures (explicitly labeled in Fig. 2) are of particular energetic stability relative to all others in the same set. This makes them thermodynamically the most likely intermediates in a dissociation process and therefore, the most likely structures to be observed in STM experiments. Figure 3 provides schematic atomic structures and Table I details the energy results for these intermediates.

Structure (A1) is the dimer-end $\mathrm{PH}_{3}$ adsorption site (as established by ab initio studies $[8,13,14]$ ) and is found to be $0.62 \mathrm{eV}$ more stable than gas-phase $\mathrm{PH}_{3}$ and the bare surface. Structure (B1) is the dissociation product discussed by Miotto et al. [14] with $\mathrm{PH}_{2}$ and $\mathrm{H}$ bound to opposite ends of a dimer. (B1) is far more stable than any other $\mathrm{PH}_{2}$ structure, including the $\mathrm{PH}_{2}$ dimer-bridge

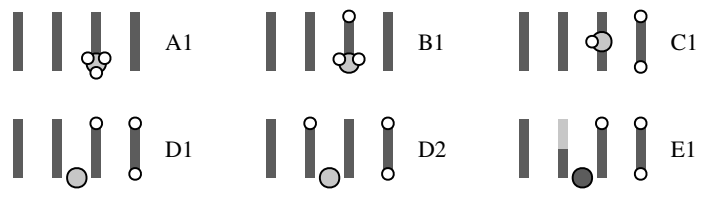

FIG. 3. Schematic plan view of structures discussed in text. P, $\mathrm{H}$, and $\mathrm{Si}$ atoms are indicated by gray, white, and black circles, respectively. Silicon dimers are indicated by solid black bars; the solid gray half bar in structure (E1) indicates the position of the dimer incorporated $\mathrm{P}$ atom. 
TABLE I. Energies of $\mathrm{PH}_{3}$ adsorption and dissociation products as indicated in Fig. 3. All energies are in eV, and are expressed relative to the corresponding (A1) structure.

\begin{tabular}{ccrrrrc}
\hline \hline \multirow{2}{*}{ Structure } & \multicolumn{2}{c}{ CPMD } & VASP & Gaussian & Site of $\mathrm{PH}_{x}$ \\
& & $4 \times 4$ & $2 \times 4$ & $2 \times 4$ & cluster & \\
\hline $\mathrm{PH}_{3}$ & (A1) & 0.00 & 0.00 & 0.00 & 0.00 & dimer end \\
$\mathrm{PH}_{2}+\mathrm{H}$ & $(\mathrm{B} 1)$ & -1.34 & -1.22 & -1.10 & -1.40 & dimer end \\
$\mathrm{PH}+2 \mathrm{H}$ & $(\mathrm{C} 1)$ & -2.22 & -2.24 & -2.00 & -2.49 & dimer bridge \\
$\mathrm{P}+3 \mathrm{H}$ & $(\mathrm{D} 1)$ & -2.45 & -2.39 & -2.35 & -2.51 & end bridge \\
& $(\mathrm{D} 2)$ & -2.42 & -2.36 & -2.34 & -2.51 & end bridge \\
$\mathrm{Si}+3 \mathrm{H}$ & $(\mathrm{E} 1)$ & -2.91 & -2.83 & -2.73 & -3.35 & incorporated \\
\hline \hline
\end{tabular}

model for the centered STM feature proposed by Lin et al. [9]. The most stable $\mathrm{PH}$ structure is (C1), which consists of a $\mathrm{PH}$ unit in a dimer-bridge site adjacent to a monohydride dimer. This structure, which contains $\mathrm{PH}$ in a three-membered ring, is $0.88 \mathrm{eV}$ more stable than the (B1) structure, previously considered to be the most stable $\mathrm{PH}_{x}$ structure $[14,18]$. The most stable structures containing a bare phosphorus atom are (D1) and (D2), which both contain the $\mathrm{P}$ atom in an end-bridge site, an adjacent monohydride dimer, and a single $\mathrm{H}$ on one of the bridged dimers. (D1) and (D2) differ only by the position of the single $\mathrm{H}$ and are, respectively, $0.23 \mathrm{eV}$ and $0.20 \mathrm{eV}$ more stable than (C1). The most stable structure overall in our survey is structure (E1) where the $\mathrm{P}$ atom is incorporated into a surface dimer and the ejected $\mathrm{Si}$ atom sits in an end-bridge site. This structure is $0.46 \mathrm{eV}$ more stable than (D1), making it $2.91 \mathrm{eV}$ more stable than (A1), the initial adsorption product of $\mathrm{PH}_{3}$. We note, however, that ejected $\mathrm{Si}$ atoms are not observed experimentally until after an anneal at $\approx 350{ }^{\circ} \mathrm{C}[10]$. This indicates that at room temperature, access to P-incorporated structures is kinetically hindered.

We now consider the STM appearance of these low energy structures and will show that structures (B1), (C1), and (D2) (Fig. 4) are in very good agreement with the asymmetric, centered, and U-shaped features seen in Fig. 1.

The asymmetric STM feature has been assigned previously [5] to the initial dissociation product $\mathrm{PH}_{2}+\mathrm{H}$
[(B1) in our notation]. The qualitative agreement of our simulated STM image of (B1) in filled and empty state [Fig. 4(a)] with the experimental images confirms this assignment. Furthermore, the single-dimer width of the asymmetric feature leaves only nondissociated $\mathrm{PH}_{3}$ [structure (A1)] as a viable alternative assignment. However, the (A1) feature produces strong pinning $\left(E_{p}=\right.$ $0.095 \mathrm{eV}$, see [19]) of the surrounding dimers, while the (B1) feature does not pin $\left(E_{p}=0.003 \mathrm{eV}\right)$. The absence of pinning in Fig. 1(b) thus supports the (B1) assignment.

We consider now the $(\mathrm{C} 1)$ structure that we assign to the observed centered feature. Figure 4(b) shows that the simulated STM image contains a centered feature arising from the $\mathrm{P}$ lone pair, which appears very bright in filled state (due to strain in the three-membered ring) and dimmer in empty state, as observed in the experimental image. The adjacent hydrogen-saturated dimer (monohydride) has a simulated STM image that is dark in filled and empty state due to well-separated $\sigma$ and $\sigma^{*}$ states. This explains the dark dimer observed adjacent to the centered feature in Fig. 1(c) and 1(f).

We attribute the U-shaped STM feature to structure (D2), shown in perspective view in Fig. 5. The bare $\mathrm{Si}$ atom in the center back of the structure view has an upwardly displaced, threefold pyramidal coordination (indicating a lone-pair orbital and negative charge at this site) that appears bright in the simulated filled-state image [Fig. 4(c)] and dim in empty state. The P atom forms a covalent bond with a second-layer $\mathrm{Si}$ atom at the expense of one of the Si-Si backbonds (Fig. 5 shows one of the backbonds is elongated to $2.56 \AA$ from typically $2.38 \AA$ ). As illustrated in the bonding diagram (Fig. 5), this would have rendered the first-layer Si atom undercoordinated (and cationic) were it not for the $\mathrm{P}$ atom that compensates by donating some of its electron density. This interaction stabilizes the P lone-pair electrons (making them dim in filled state) and creates an antibonding orbital that images brightly in empty state. Finally, the monohydride and isolated hydrogen appear dark in filled and empty state as for (C1). Together, these components produce simulated STM images [Fig. 4(c)] that reproduce
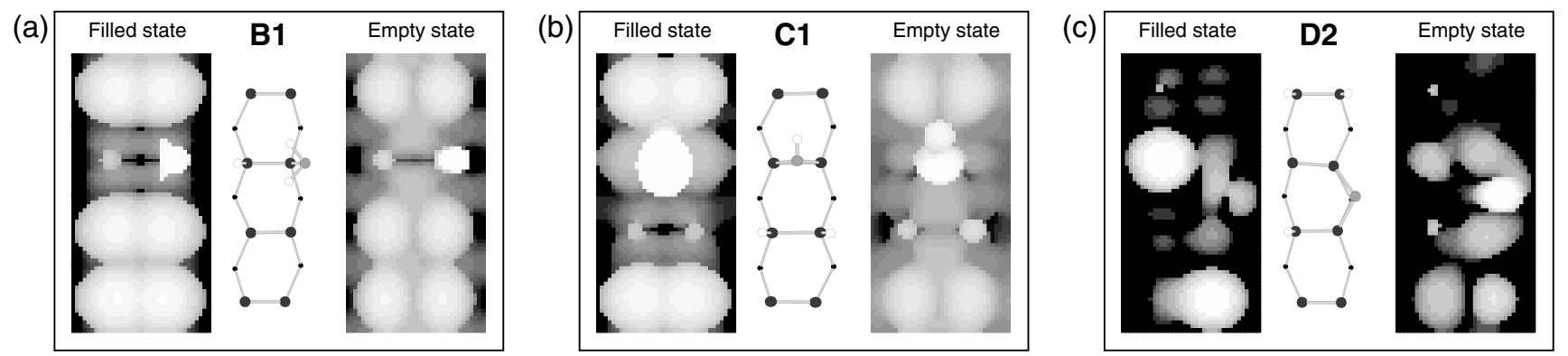

FIG. 4. Simulated filled- and empty-state STM images of (a) structure (B1), (b) structure (C1), and (c) structure (D2), which we assign to the experimentally observed asymmetric, centered, and U-shaped STM features seen in Fig. 1. Because of the absence of pinning, the images for (B1) and (C1) are averaged over both buckling isomers for the bare dimers [19]. Images were calculated for a $(2 \times 4)$ slab model in the Tersoff-Hamann approximation [20] summing over the four states nearest the Fermi energy. 


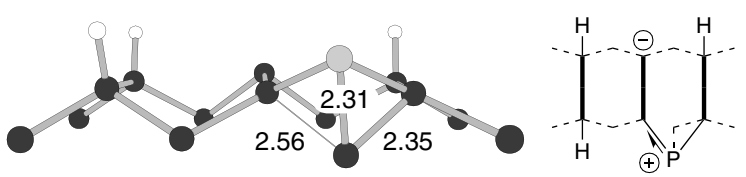

FIG. 5. 3D view and schematic bonding diagram of structure (D2). $\mathrm{P}, \mathrm{H}$, and $\mathrm{Si}$ atoms are indicated by gray, white, and black spheres, respectively. Bond lengths (given in $\AA$ ) illustrate the lengthening of a Si-Si backbond.

the characteristic U-shaped appearance seen experimentally in Fig. 1(d) and 1(g). We note further that Si dimers adjacent to the U-shaped feature in Fig. 1(d) are pinned (see [19]) on one side of the defect only. This is consistent with structure (D2) for which pinning is predicted on the isolated hydrogen side of the defect $\left(E_{p}=0.022 \mathrm{eV}\right)$, but not on the monohydride side $\left(E_{p}=0 \mathrm{eV}\right)$.

The relationship between the three STM features in the dissociation process is illustrated by the time sequence of images in Figs. 6(a)-6(c), which show the asymmetric feature (B1) converting first to a centered feature (C1) and subsequently to a U-shaped feature (D2). These conversions are routinely observed in our STM experiments [11], but the reverse processes are never seen. This sequence progresses towards increasing energetic stability according to Table I. We can thus outline (Fig. 6) a mechanism for $\mathrm{PH}_{3}$ dissociation on the $\mathrm{Si}(001)$ surface in which adsorbed $\mathrm{PH}_{3}$ (A1) dissociates via proton shift to the asymmetric feature (B1). This in turn stabilizes to the centered feature $(\mathrm{C} 1)$, which undergoes further stabilization to the U-shaped feature (D2), most likely by another proton transfer followed by a shift of the $\mathrm{P}$ atom from the dimer-bridge to the end-bridge site. Further, on account of the broken backbond, we consider (D2) to be a plausible point of entry for the thermally-induced incorporation of $P$ into a surface dimer.

In summary, we have performed an extensive ab initio survey of dissociation products of $\mathrm{PH}_{3}$ on the $\mathrm{Si}(001)$
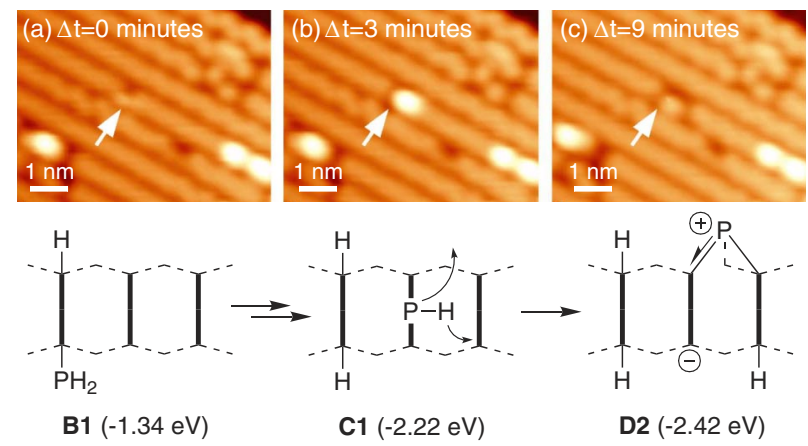

FIG. 6 (color online). STM images showing the progressive dissociation of $\mathrm{PH}_{3}$ via (a) the asymmetric feature (B1), (b) the centered feature (C1), and (c) the U-shaped feature (D2). All images were acquired with $-2.0 \mathrm{~V}$ sample bias and $0.2 \mathrm{nA}$. The schematic outlines the observed mechanism of dissociation. surface. On the basis of energetics and simulated STM images, we have assigned three common STM features observed on the surface after dosing with $\mathrm{PH}_{3}$. This assignment identifies for the first time a detailed pathway for the complete dissociation of $\mathrm{PH}_{3}$ and the incorporation of $\mathrm{P}$ into the $\mathrm{Si}(001)$ surface. Knowledge of this mechanism closes an important gap in the understanding of the P-doping process of silicon. An understanding of this mechanism is of particular utility to the fabrication of novel nanoelectronic devices, which relies on the atomically precise placement and controlled dissociation of single $\mathrm{PH}_{3}$ molecules on the $\mathrm{Si}(001)$ surface [5].

This work was supported by the Australian Partnership for Advanced Computing.

[1] G. L. Snider et al., J. Appl. Phys. 85, 4283 (1999).

[2] J. R. Tucker and T.C. Shen, Int. J. Circuit Theory Appl. 28, 553 (2000).

[3] B. E. Kane, Nature (London) 393, 133 (1998).

[4] J. L. O'Brien et al., Phys. Rev. B 64, 161401(R) (2001).

[5] S. R. Schofield et al., Phys. Rev. Lett. 91, 136104 (2003).

[6] Y. Wang, M. J. Bronikowski, and R. J. Hamers, J. Phys. Chem. 98, 5966 (1994).

[7] Y. Wang, X. Chen, and R. J. Hamers, Phys. Rev. B 50, 4534 (1994).

[8] L. Kipp et al., Phys. Rev. B 52, 5843 (1995).

[9] D.-S. Lin, T.-S. Ku, and T.-J. Sheu, Surf. Sci. 424, 7 (1999).

[10] N. J. Curson et al. Phys. Rev. B 69, 195303 (2004).

[11] N. J. Curson, et al. (to be published).

[12] M. L. Yu and B. S. Meyerson, J. Vac. Sci. Technol. A 2, 446 (1984).

[13] J. Shan, Y. Wang, and R. J. Hamers, J. Phys. Chem. 100, 4961 (1996).

[14] R. Miotto, G. P. Srivastava, and A. C. Ferraz, Phys. Rev. B 63, 125321 (2001); Surf. Sci. 482-485, 160 (2001).

[15] J. Hutter et al. computer code CPMD, MPI für Festkörperforschung and IBM Zürich Research Laboratory, 1995-2004.

[16] G. Kresse and J. Hafner, Phys. Rev. B 47, R558 (1993); ibid. 49, 14251 (1994); G. Kresse and J. Furthmüller, Phys. Rev. B 54, 11169 (1996).

[17] M. J. Frisch et al., Gaussian 03, Revision B.03 (Gaussian, Inc., Pittsburgh PA, 2003).

[18] N. Maity, L.-Q. Xia, S. E. Roadman, and J. R. Engstrom, Surf. Sci. 344, 203 (1995).

[19] At room temperature (RT), Si dimers appear symmetric in STM filled-state images because of the rapid thermal flipping between two buckled isomers. Pinning, that is, the buckled appearance of Si dimers adjacent to a surface feature, results when one isomer becomes energetically favored due to coupling with the feature. When the energy difference $\left(E_{p}\right)$ between the isomers is significant relative to $k T(0.025 \mathrm{eV}$ at $\mathrm{RT})$, pinning is observed.

[20] J. Tersoff and D. R. Hamann, Phys. Rev. Lett. 50, 1998 (1983). 